\title{
Effects of near-road and regional air pollution: the challenge of separation
}

\author{
Nino Künzli ${ }^{1,2}$
}

As people spend most of their lifetime at home, outdoor air pollution levels near the home remain an important determinant of personal exposure to pollution from outdoor sources. With every breath, we inhale a complex mixture of hundreds of such pollutants. A simplified categorisation of these pollutants distinguishes local, freshly emitted (primary) pollutants from regional, aged (secondary) pollutants. To understand people's exposure to the latter-more homogeneously distributed pollutants (eg, fine particles, PM2.5 or ozone)-many studies use data from fixed-site monitoring stations, considered to be representative of exposure in the entire community. For near-road local types-such as diesel soot, ultrafine nanoparticles, NO or $\mathrm{CO}$-this approach is insufficient. $^{1}$ Freshly emitted pollutants from vehicle exhaust and resuspended particles show strong spatial gradients, with concentrations exponentially decreasing, reaching within $50-500 \mathrm{~m}$ of busy roads $10-30 \%$ of the levels observed on the street. $^{2}$ Thus, to understand exposure to these locally emitted pollutants, we need to use small-scale local measurements and models.

The main argument for making this distinction is that regional and near-road traffic-related pollutants may have different health effects. The literature on childhood asthma and air pollution supports this notion and reveals two consistent patterns: (1) comparison of the (multivariate-adjusted) asthma prevalence or incidence across communities with different air quality does not support the hypothesis that regional air pollutants play a relevant aetiological role $^{3}$; (2) studies estimating exposure to near-road traffic-related pollutants indicate that children growing up close to busy roads have a higher risk of developing asthma than those living a few hundred metres away. ${ }^{4} 5$ The Southern Californian Children's Health Study (CHS) is one of several epidemiological studies that recognises the asthmogenic

\footnotetext{
${ }^{1}$ Swiss Tropical and Public Health Institute, Basel,

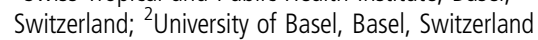

Correspondence to Professor Nino Künzli, Department of Epidemiology and Public Health, Swiss Tropical and Public Health Institute, Socinstrasse 57, PO Box, Basel 4002, Switzerland; Nino.Kuenzli@unibas.ch
}

role of near-road pollutants, ${ }^{67}$ confirming biologically plausible models. ${ }^{8}$

Landmark cohort studies initiated by the CHS team provide both crosssectional and longitudinal evidence for regional ambient air pollution affecting lung growth during adolescence. ${ }^{9}{ }^{10}$ The question of an independent role of nearroad traffic-related air pollution was a later subject of analyses in two CHS cohorts, recruited in 1993 and $1996 .{ }^{11}$ In those analyses-and in contrast with the asthma findings-lung function growth was associated with both regional pollutants and residential distance from highways as a marker of near-road pollution.

CHS researchers have explored the hypothesis that near-road and regional pollutants play a distinct role in the level of lung function among a cohort of 57-year-old children, recruited in 20022003. ${ }^{12}$ In the new study, near-road pollutants were estimated for each child, linking residential location with land-use regression models and using $\mathrm{NO}, \mathrm{NO}_{2}$ and NOx as markers of near-road pollution. Exposure to regional pollution relied on fixed-site monitor data, available in each community. Lung function levels were significantly associated with both the near-road and community mean levels of pollution. The coefficients observed for $\mathrm{NO}_{2}$, listed as a marker for both nearroad and regional pollution, indicate that the effects of near-road exposure were much stronger than those attributed to regional pollutants. With PM2.5 as a marker for regional pollution, regional effects were statistically significant.

Thus, for health outcomes such as lung function, where possibly both the nearroad and regional secondary pollutants matter, the question then arises of whether we can investigate, separate and quantify these effects independently. First, this is of scientific interest, as these groups of pollutants have somewhat different characteristics that may act through distinct pathways. For example, the nano-sized ultrafine particle fractions-of particular relevance near roads-have some toxic properties and effects, such as possibly direct transitions into the brain, for example, that are unlikely to be relevant for larger particles. Second, the distinction is also relevant for policy makers and risk assessors to more comprehensively target and quantify the burden of disease attributable to pollution and related clean air interventions. ${ }^{13}$ While both near-road and regional pollutants are very heavily driven by traffic in the Southern Californian region, this is not universally true. Moreover, whereas some near-road pollution problems may be solved by local action and tackled by local authorities (eg, retrofitting buses with particle filters), regional pollutants require regional and transnational action.

The CHS, with its multi-community design, provides a setting with sufficient contrasts in regional pollution across communities and in near-road pollutants within communities. For each subject, Urman et al determine the extent to which the home-outdoor NOx concentration deviates from the community mean level to generate within-community exposure estimates that do not correlate with the between-community contrasts. ${ }^{12}$ Although one interpretation of their findings is, indeed, that local pollutants result in independent effects, it must be emphasised that regional pollutant exposure relied on fixed-site monitor data alone. Thus, one may hypothesise that the finding of 'independent associations' is an artefact of the analytical approach. Imagine that, instead of testing the air pollution hypotheses, we are testing the association of pack-years of smoking with lung function in adults in a 10 -community study. Let us pretend that the distribution of near-road pollutants shown in figure $1 \mathrm{~B}$ of Urman et $a l^{12}$ does not reflect residential $\mathrm{NO}_{2}$ but the distribution of packyears of cigarettes within each community. Let us further assume that we analysed the data in the same way, namely to show how the individual deviates from the community-mean pack-years. What would the association between pack-years and lung function look like for the withinand between-community estimates? In theory, one should expect the same packyears coefficients for both the withincommunity and the between-community effects of smoking. The price we pay for such an analytical approach is some loss of statistical power, but the gain is the removal of unmeasured spatial confounders possibly related to 'communities'-a major concern in air pollution research. Moreover, the between-community estimate may be subject to measurement error, as it is not an individually assigned exposure. This point may be particularly relevant in an air pollution study such as that of Urman et al, where the regional fixed-site monitor value may be biased 
toward null, as the fixed-site monitor concentration was not equally representative of the community-specific, populationweighted, residential mean $\mathrm{NO}_{2}$ exposures. This may, in part, explain why the coefficient for regional $\mathrm{NO}_{2}$ was some four times smaller (and not significant) than the near-road $\mathrm{NO}_{2}$ effect estimate. The between-community estimates were, however, significant for more homogeneously distributed particles. As these estimates did not correlate closely with the fixed-site monitor levels of $\mathrm{NO}_{2}$, one may again endorse the interpretation of independent effects. However, these regional pollutants may also vary within communities, which was not taken into account with the fixed-site monitor approach, thus the true correlation between nearroad and regional pollution remains unknown.

In light of such uncertainties, one question remains: what should one do to convincingly disentangle and quantify the long-term effects of both near-road and regional pollutants? I think we need studies where both the regional and nearroad pollutants are estimated at the individual level, thereby negating the sole use of fixed-site monitor data to represent exposure to 'regional pollution'. Some studies have gone down this avenue, ${ }^{14}$ and, most recently, the European ESCAPE network adopted fully standardised measurements and land-use modelling approaches to individually assign homeoutdoor (residential) estimates of seven markers of ambient air pollution to participants in more than 30 cohorts. ${ }^{15}$ Markers ranged from indicators of secondary regional pollutants $(\mathrm{PM} 2.5)^{16}$ to local near-road markers (NOx or reflectance of PM2.5-indicating local diesel soot). As shown in ESCAPE, the approach may also face limits, as the contrasts between regional pollutants may be inherently low within small study areas. For example, for PM2.5, the ratio between street site and urban background levels was as low as 1.02 and as high as 1.3 among ESCAPE regions, but was usually higher-up to 1.77-for the near-road marker of PM2.5 reflectance. ${ }^{16}$ In a recent analysis based on six childhood cohorts, $\mathrm{NO}_{2}, \mathrm{NOx}, \mathrm{PM} 2.5$ absorbance and PM2.5 (but not PM10, PM coarse or markers of neighbourhood traffic) were all associated with significantly lower lung function in 6-8-year-old children. ${ }^{17}$ Two-pollutant models for local $\left(\mathrm{NO}_{2}\right)$ and regional (PM2.5) pollutants were challenged by multi-collinearities and thus did not conclusively support the notion of independent effects.
Mega-cohorts in very large regionsfor example, in mega-cities in low-income economies with far more diverse and different sources of pollutants-may provide the setting for such individual approaches. For example, a land-use regression model for the mega-city of Tehran (Iran), developed for an area of $30 \times 30 \mathrm{~km}$, revealed very different patterns, with severalfold contrasts for both near-road traffic-related and (possibly) regional pollutants (PM10, $\mathrm{NO}_{2}, \mathrm{SO}_{2}$ ) (publication in preparationpresented at the 2013 conference). The rather low spatial correlations among these pollutants provide a substantially different setting. Modelling both nearroad and regional pollutants at the individual level will be needed to understand the specific long-term effects of near-road ultrafine particles.

In sum, while the landmark CHS research indicates independent effects of near-road and regional pollutants on lung function, this may not be generalisable. Further research is needed to better understand the public health role of different sources and characteristics of ambient air pollution. Whereas fixed-site monitor data remain very important for policy making and research using timeseries analyses, trend assessment, calibration and modelling, epidemiological studies investigating the long-term effects of air pollution should not rely on fixedsite monitor data alone, but estimate exposure for both regional and local clusters of pollutants at the individual level. This strategy will also open doors to allow us to disentangle all health-relevant environmental stressors of traffic, namely primary and secondary exhaust-related and resuspended pollutants as well as noise, which remain a challenge to be separated and tackled. ${ }^{18} 19$

\section{Competing interests None.}

Provenance and peer review Not commissioned; internally peer reviewed.

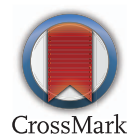

To cite Künzli N. Thorax 2014;69:503-504.

Published Online First 3 January 2014

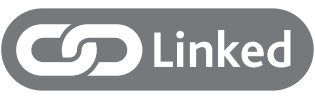

- http://dx.doi.org/10.1136/thoraxjnl-2012-203159

Thorax 2014;69:503-504.

doi:10.1136/thoraxjnl-2013-204991

\section{REFERENCES}

1 Health Effects Institute. Traffic-Related Air Pollution: a Critical Review of the Literature on Emissions, Exposure, and Health Effects. Boston: HEl, 2009; Special Report 17.

2 Zhu Y, Hinds WC, Kim S, et al. Concentration and size distribution of ultrafine particles near a major highway. J Air Waste Manag Assoc 2002;52:1032-42. PubMed PMID: Department of Environmental Health Sciences, University of California, Los Angeles 90095, USA.

3 Anderson R, Favarato G, Atkinson R. Long-term exposure to outdoor air pollution and the prevalence of asthma: meta-analysis of multi-community prevalence studies. Air Qual Atmos Health 2013;6:57-68.

4 Anderson R, Favarato G, Atkinson R. Long-term exposure to air pollution and the incidence of asthma: meta-analysis of cohort studies. Air Qual Atmos Health 2013;6:47-56.

5 Salam MT, Islam T, Gilliland FD. Recent evidence for adverse effects of residential proximity to traffic sources on asthma. Curr Opin Pulm Med 2008;14:3-8.

6 McConnell R, Islam T, Shankardass K, et al. Childhood incident asthma and traffic-related air pollution at home and school. Environ Health Perspect 2010;118:1021-6.

7 McConnell RB, Berhane K, Yao L, et al. Traffic, susceptibility, and childhood asthma. Env Health Perspect 2006;114:766-72.

8 Nel A. Atmosphere. Air pollution-related illness: effects of particles. Science 2005;308:804-6.

9 Gauderman WJ, Avol E, Gilliland F, et al. The effect of air pollution on lung development from 10 to 18 years of age. N Engl J Med 2004;351:1057-67.

10 Peters JM, Avol E, Gauderman WJ, et al. A study of twelve Southern California communities with differing levels and types of air pollution. II. Effects on pulmonary function. Am J Respir Crit Care Med 1999;159:768-75.

11 Gauderman WJ, Vora H, McConnell R, et al. Effect of exposure to traffic on lung development from 10 to 18 years of age: a cohort study. Lancet 2007;369:571-7.

12 Urman R, McConnell R, Islam T, et al. Associations of children's lung function with ambient air pollution: joint effects of regional and near-roadway pollutants. Thorax 2014;69:540-7.

13 Henschel S, Atkinson R, Zeka A, et al. Air pollution interventions and their impact on public health. Int $J$ Public Health 2012;57:757-68.

14 Künzli N, Bridevaux PO, Liu S, et al. Traffic-related air pollution correlates with adult-onset asthma among never-smokers. Thorax 2009;64:664-70.

15 Wang M, Beelen R, Basagana X, et al. Evaluation of land use regression models for NO2 and particulate matter in 20 european study areas: the ESCAPE project. Environ Sci Technol 2013;47:4357-64.

16 Eeftens M, Beelen R, de Hoogh K, et al. Development of land use regression models for PM(2.5), PM(2.5) absorbance, PM(10) and PM (coarse) in 20 European study areas; results of the ESCAPE project. Environ Sci Technol 2012;46:11195-205.

17 Gehring U, Gruzieva O, Agius RM, et al. Air pollution exposure and lung function in children: the ESCAPE project. Environ Health Perspect 2013;121:1357-64.

18 Foraster M. Is it traffic-related air pollution or road traffic noise, or both? Key questions not yet settled! Int J Public Health 2013;58:647-8.

19 Tetreault LF, Perron S, Smargiassi A. Cardiovascular health, traffic-related air pollution and noise: are associations mutually confounded? A systematic review. Int J Public Health 2013;58:649-66. 